\title{
«La référence scolaire : de la figuration littéraire aux modèles langagiers, l'exemple de Giono »
}

"The academic reference: from literary figuration to language models, the example of Giono"

Sophie Jollin-Bertocchi

\section{(2) OpenEdition}

Journals

Édition électronique

URL : https://journals.openedition.org/pratiques/10805

DOI : $10.4000 /$ pratiques. 10805

ISSN : 2425-2042

Éditeur

Centre de recherche sur les médiations (CREM)

Référence électronique

Sophie Jollin-Bertocchi, « « La référence scolaire : de la figuration littéraire aux modèles langagiers, l'exemple de Giono » », Pratiques [En ligne], 191-192 | 2021, mis en ligne le 15 décembre 2021, consulté le 05 janvier 2022. URL : http://journals.openedition.org/pratiques/10805 ; DOI : https://doi.org/ 10.4000/pratiques. 10805

Ce document a été généré automatiquement le 5 janvier 2022.

(c) Tous droits réservés 


\section{«La référence scolaire : de la figuration littéraire aux modèles langagiers, l'exemple de Giono »}

"The academic reference: from literary figuration to language models, the example of Giono"

\section{Sophie Jollin-Bertocchi}

1 La « mise en relation des pratiques d'écriture littéraires avec les exercices et la vulgate scolaires " (Philippe, 2003, p. 379) ${ }^{1}$ n'étant plus à démontrer, il reste à observer sa singularité au sein des œuvres, son degré, ses modalités et sa valeur ${ }^{2}$. Explicitement ou implicitement, l'École est saisie au travers du prisme de l'imaginaire d'un écrivain. Sa représentation dans la fiction est extrêmement variable, très marquée chez certains auteurs, absente chez d'autres - dans ce dernier cas c'est donc à partir du seul style littéraire que peut s'appréhender le rapport aux modèles scolaires-, valorisée positivement ou négativement. Plus exactement, c'est la relation entre la figuration (ou non) de l'École dans l'œuvre et les formes langagières qui permet de construire la référence scolaire telle qu'envisagée par un écrivain. À partir du deuxième tiers du XIX ${ }^{\mathrm{e}}$ siècle, cette référence est double en réalité, elle recouvre un enseignement primaire destiné à l'instruction du peuple, dominé par un idéal de simplicité de la langue, opposé à un enseignement secondaire destiné à la formation des élites et caractérisé par une plus grande expressivité langagière (Balibar, 1974). Ces deux «ordres» de formation étaient bien concurrents et non successifs. Les écrivains formés au cours de cette période devaient-ils alors nécessairement choisir leur camp? Si l'institution scolaire a pu être mise à distance, voire reniée par quelques auteurs avant-gardistes ou autodidactes, il parait délicat de s'abstraire de la perspective consistant à « voir dans le rapport autodidacte à la culture et dans l'autodidacte lui-même des produits du système scolaire, seul habilité à transmettre ce corps hiérarchisé d'aptitudes et de savoirs qui constitue la culture légitime et à consacrer, par l'examen et les titres, l'accès à un niveau déterminé d'initiation» (Bourdieu, 1979, p. 378). Tandis que les fictions "postmodernes ", à partir des années 1950, mettent ostensiblement en question la soumission à la norme scolaire, quelle qu'elle soit, le rejet des modèles langagiers 
transmis par l'École - sans doute à distinguer du rejet de l'institution elle-même semble difficilement défendable s'agissant des œuvres fictionnelles de la première moitié du $\mathrm{xx}^{\mathrm{e}}$ siècle, dont l'écriture, en dépit d'une part d'innovation chez certains auteurs, se rattache encore à la sphère du lisible et du bien écrit. Ainsi cette étude portera sur un écrivain doublement représentatif du premier tiers $d u x^{e}$ siècle, J. Giono, d'une part du fait qu'il a été scolarisé au tout début du siècle, soit au moment où s'achève l'institutionnalisation de l'École républicaine en France, et d'autre part parce qu'il a poursuivi sa formation littéraire en autodidacte après avoir interrompu ses études au lycée, incarnant à ce titre la liberté vis à vis de la culture scolaire qui caractérise les premières décennies $\mathrm{du} \mathrm{xx}^{\mathrm{e}}$ siècle, en réaction à cette institutionnalisation ${ }^{3}$. J'essaierai de comprendre le rapport problématique que J. Giono entretient avec l'École à travers les modalités de la construction de la référence scolaire. Après avoir présenté les éléments de la "posture » (Meizoz, 2007) de semiautodidacte adoptée par J. Giono, j'interrogerai donc la présence de l'École dans son œuvre à travers sa figuration dans la fiction et les formes langagières, en me centrant sur les patrons phrastiques.

\section{Une posture de semi-autodidacte}

2 Né en 1895, J. Giono quitte l'enseignement secondaire en octobre 1911, au début de la classe de première ${ }^{4}$, à l'âge de seize ans, contraint de chercher un travail par la dégradation de l'état de santé de son père. C'est bien au-delà de l'obligation légale de l'époque, car en France, depuis la loi «Jules Ferry» de 1882, si l'instruction était obligatoire jusqu'à treize ans révolus, les élèves titulaires du certificat d'études primaires pouvaient quitter l'École plus tôt. Les études secondaires n'étant pas gratuites, les enfants de milieu modeste la quittaient de bonne heure; les élèves présentant des dispositions scolaires avaient cependant la possibilité de poursuivre leurs études grâce à une bourse. J. Giono, lui, «bénéficie du collège gratuit de Manosque (situation qui le place dans un rôle proche de celui de boursier d'origine modeste, comme Péguy ou Guéhenno)» (Meizoz, 2001, p. 324), ou encore A. Camus et M. Pagnol. Le collège de Manosque est «l'un des rares établissements gratuits de France, à l'époque » (Ibid., p. 325). L'École lui permettait d'espérer de meilleures conditions de vie, perspective non négligeable si l'on en juge d'après le récit autobiographique de sa jeunesse Jean le Bleu, dominé par la représentation de la misère. Il était cependant un élève plutôt indifférent à l'École, et non mécontent de la quitter, d'après ses propres déclarations. De fait un certain nombre de fautes d'orthographe émaillent les manuscrits de l'écrivain... Une chose est sure, la figuration de l'institution scolaire est quasiment absente de l'œuvre gionienne, contrairement à ce que l'on observe dans l'œuvre d'autres auteurs de la même époque cités plus haut, M. Pagnol notamment, né la même année que J. Giono, ou bien encore Colette et R. Martin du Gard.

3 La revendication d'une formation scolaire tronquée, posture escortée de la valeur de liberté, était dans l'air du temps 5 . Il est néanmoins difficile de comparer le parcours de J. Giono à celui d'un autodidacte ou d'un élève de l'École primaire. Lycéen peu brillant, quoiqu'il adopte une posture critique vis-à-vis du secondaire, sa formation est bien celle d'un élève du secondaire. C'est donc seulement après avoir quitté le lycée que les 
voies d'accès à la culture empruntées par J. Giono s'avèrent conformes à la définition de connaissances qui ne sont garanties par aucun titre ni par personne :

[...] les définitions usuelles de l'autodidacte et de l'autodidaxie (celles des dictionnaires), restreignent l'usage de ces notions à des modalités spécifiques d'acquisition de l' «instruction » [...]. Toutes insistent sur l'auto-apprentissage, l'apprentissage solitaire [...]. Aucune des définitions usuelles ne fait explicitement référence au système scolaire: sans doute parce que l'apparition du terme (1580) est bien antérieure à l'obligation scolaire [...]. (Poliak, 1992, p. 29-30)

Il s'agit à présent de confronter les données externes exprimant la position de l'auteur vis-à-vis de l'institution scolaire avec les données internes à l'œuvre fictionnelle.

\subsection{Les données externes}

$5 \quad$ Rappelons les éléments biographiques :

À cinq ans, il a été mis à l'école. Sa mère, très pieuse, insiste pour qu'il aille chez les sœurs Présentines : il transposera dans Jean le Bleu les souvenirs merveilleux qu'il en a conservés. Deux ans plus tard, il passe au petit collège de Manosque, où il restera neuf ans, jusqu'à la fin de la seconde, mais dont il ne dira jamais rien ; aucun de ses professeurs ne le remarque ; bon élève sans excès, il obtient assez régulièrement, et sans grand mal vu le peu de concurrence, des prix [...]. (Citron, 1995, p. 12)

6 L'enchantement des débuts scolaires aurait cédé la place à l'indifférence, attitude qui peut se justifier par les origines sociales modestes et l'enracinement régional de l'écrivain :

Quand il se définit en provincial heureux de l'être et refuse l'identité d'homme de lettres, le premier Giono actionne la thématique anti-urbaine, et son corrélat intellectualiste. Ces thèmes rejoignent tantôt la haine du bourgeois qui caractérise les intellectuels communistes, tantôt les positions ultra-régionalistes opposées à la centralisation parisienne. (Meizoz, 2001, p. 326)

7 J. Meizoz insiste sur l'influence déterminante de l'identité populaire, rurale et régionale de J. Giono, résumant son rapport à l'École au «français non-académique », au « topos anti-grammairien " (Meizoz, 2001, p. 340-342). La position régionaliste et la position anti-intellectualiste, communes à d'autres écrivains de l'époque aux origines sociales semblables, créent les conditions de la posture d'autodidacte, qui se situe à la limite de la provocation dans une société française encore dominée par le modèle du grand écrivain, même si ce statut «n'a cessé de se dégrader du début du siècle à la Seconde Guerre mondiale » (Philippe, 2003, p. 386). J. Giono n'a pas mâché ses mots à propos de ses années d'enseignement secondaire :

J. Amrouche - Vous serait-il possible de nous parler de ces années de collège, est-ce qu'elles ont eu une grande importance pour vous?

J. Giono - Non, pas très grande, je suis allé au collège comme tout le monde y va [...]. Ça n'a laissé absolument aucune trace. J'ai commencé, si l'on peut employer le mot « cultiver », j'ai commencé à me cultiver quand la curiosité s'est éveillée en moi. Au collège, je n'ai jamais eu de curiosité. Après avoir quitté le collège, j'ai commencé à être curieux parce que j'étais libre. L'importance, pour moi, c'était d'être libre de choisir. À ce moment-là, je me suis mis à choisir, avec discernement ou sans discernement, ça n'a aucune importance, mais librement. (Giono, 1990, p. 133)

8 L'écrivain fournit ainsi une explication d'ordre psychologique, à savoir la difficulté à supporter les contraintes scolaires, topos lié à la posture d'autodidacte. Dans un autre entretien, il revient sur cette idée de liberté en l'associant à la notion de plaisir, et 
emploie le terme « autodidacte » même pour caractériser sa formation à la littérature et à la culture :

J'ai toujours vu que pour atteindre à une agrégation, qui est l'entrée des grands professeurs, [...] il fallait s'imposer une discipline telle que ça mettait des œillères, ça bouchait la vue et ça détournait l'odorat de certaines choses; on était obligé de mettre le nez dans certaines pistes, de ne pas les quitter, alors, là, évidemment, il y a certainement un danger pour le professeur. Et certainement là une plus grande liberté, une plus grande facilité pour l'autodidacte. Ce qui, en tout cas, est en faveur de l'autodidacte, c'est qu'il éprouve beaucoup de plaisir, il prend beaucoup de plaisir à se cultiver, il n'est pas ennuyé, la culture n'arrive pas chez lui comme une matière étrangère, il la désire avant de la prendre, et il digère parfaitement bien. (Carrière, 2000, p. 151)

Une idéalisation perce peut-être à travers la clausule hyperbolique de la citation. Cette déclaration est en effet paradoxale, car en contrepoint de l'allégresse suscitée par l'absence de contraintes, la démarche autodidacte relève de l'exploration d'un labyrinthe, et à ce titre est susceptible de créer un effet paralysant ou de s'avérer d'une efficacité relative : «Le fait de s'instruire, pour l'autodidacte, ne va jamais de soi. S'il choisit de le faire, souvent au prix de difficultés considérables, c'est pour des raisons fortes et qui lui sont propres » (Biron, 2007, p. 70).

En tant que père, J. Giono ne semble pas avoir tenu le même discours à ses enfants, comme en témoigne cette anecdote rapportée par sa fille ainée, Aline, dans un court récit autobiographique intitulé La composition de français :

Tout a commencé avec la mauvaise note qu'il a obtenue à son devoir de français. Ou plutôt devoir de français de ma sœur, qui avait eu l'imprudence, la malheureuse, de lui demander des conseils.

- J'en ai assez, moi, des «dont auxquels" et des "par rapport à quoi » qui remplissent tes rédactions, a répondu papa. Pour bien écrire, c'est facile : un sujet, un verbe, un complément ; un sujet, un verbe, un complément. Tu n'as pas à sortir de là. D'ailleurs, je vais te montrer, et, pour une fois, je vais te le faire moi-même, ce devoir, ça ira plus vite, et tu verras le résultat !

Et le résultat, c'est qu'il a eu 12 ํㅡ, avec cette appréciation de la maîtresse : « Un peu mieux que d'habitude ".

Ce n'était pas mal, après tout, pour quelqu'un qui a quitté l'école depuis si longtemps, et je ne comprends pas du tout pourquoi il l'a tellement mal pris, pire encore que lorsqu'il rate un de mes devoirs de calcul (et pourtant il est très fort en géométrie).

Le fait est qu'à table il s'est mis à raconter des histoires terribles sur l'école et sur les professeurs - de français en particulier.

Je lui ai répondu que s'il avait eu 18, il les aurait trouvés très bien, et il m’a dit de me taire, et je ne l'ai pas fait, et c'est comme ça que nous avons eu des mots. (Giono A., 1987, p. 107-108)

11 J. Giono s'affirme fortement attaché à la doxa scolaire élémentaire qui prône la simplicité syntaxique, en accord avec l'âge de l'élève. Sa sensibilité au sujet de l'École touche par ailleurs à la susceptibilité, qui éclate dans l'expression «des histoires terribles sur l'école et sur les professeurs", et qui intrigue. Il manifeste en réalité simultanément deux attitudes contradictoires, un respect explicite pour le savoir enseigné, qu'il s'enorgueillit de posséder, mais également, en filigrane, une hostilité vis-à-vis de l'institution scolaire. Voyons ce qu'il en est de cette tension à l'examen des figurations de l'École dans la fiction. 


\subsection{La figuration de l'École dans les fictions autobiographiques}

12 L'École est très discrètement thématisée dans l'œuvre de J. Giono. Le deuxième chapitre de Jean le Bleu (Giono, 1972a, p. 3-186), récit écrit en 1934, s'ouvre par une évocation de l'École élémentaire sur un mode oblique puisqu'il y est d'abord question du trajet de la maison à l'école, investi d'une dimension initiatique d'un autre ordre : «J'allais à l'école chez les sœurs de la Présentation. C'étaient généralement des ouvrières de ma mère qui m'y conduisaient » (Ibid., p. 11). Suit un long développement sur ces accompagnatrices qui éclipsent le lieu de destination. Dans un second temps, l'évocation du savoir et des apprentissages qui sont au cœur du monde scolaire demeure largement métonymique puisque J. Giono évoque d'abord le jardin de l'école (c'est-à-dire de nouveau un lieu extérieur à celui où se tient l'enseignement proprement dit) en une description où se déploie la métaphore filée du fruit mûr :

Le jardin de notre école était pareil à un gros fruit plein de chair et de jus. Les murs qui le pressaient le faisaient jaillir et bouillonner; il en coulait des lilas de partout ; les grands buis éclaboussaient d'ombres et d'odeurs les murs de notre petite classe et le lierre écumant d'abeilles bavait comme de la mousse de confiture du haut mur de la terrasse. (Ibid., p. 14)

Dans Jean le Bleu, l'école est donc buissonnière au sens propre, en une vision surdéterminée par le style saturé d'images de cette séquence, proche - par son sujet et sa simplicité syntaxique - du pastiche de la rédaction scolaire dans les petites classes, mais s'en démarquant par des métaphores originales. Il est ensuite question de la Sœur Dorothée et des plaisirs sensuels qui occultent totalement les préoccupations scolaires. L'École/école n'est à ce stade perçue que de l'extérieur, depuis une "cachette sous un gros laurier-rose ", de manière fugitive et sur un mode d'appréhension purement sensoriel :

Nous restions là en respirant à peine. Là-bas, la cour carrelée de grandes dalles sonnait sous les courses et les jeux; les murs grésillaient de toute une friture de cris ; on entendait sonner les anneaux de fer du portique et les câbles du trapèze qui grinçaient dans leurs crochets. Dans la petite classe, trois punis épelaient la leçon à voix égale. (Ibid., p. 15)

Les activités d'enseignement ne sont pas même mentionnées, seules la récréation, moment de divertissement et de liberté, ainsi que la punition sont dénotées, formant antithèse. De plus, l'isolement du monde scolaire permet de mettre à distance le monde extérieur ; mais c'est surtout la silhouette de l'institutrice qui captive le narrateur :

Mais, à des moments de notre classe du matin, quand, bien séparés du monde bruyant de la rue et de la ville, nous entendions le calme du couvent couler en nous avec ses pépiements de pigeons et le frottement de ses lilas contre les murs, sœur Clémentine se mettait à marcher. [...] Je n'ai jamais goûté de joie plus pure, plus musicale, plus entière, plus sûrement fille de l'équilibre que la joie de voir marcher sœur Clémentine. (Ibid., p. 17) Ce n'est donc nullement l'acquisition des connaissances, totalement passée sous silence, qui procure à J. Giono le comble de la félicité, loin de là. La référence à l'École, posée explicitement par le terme générique au début de l'évocation, s'avère déceptive. Un rapport détaché, plutôt que subversif, et un intérêt décalé, mais fondateur, sont ainsi posés.

Dans Mort d'un personnage (1948), roman qui est également d'inspiration autobiographique, se lit une autre transposition de l'expérience scolaire, précédée de manière analogue d'un récit descriptif périphérique du trajet accompagné vers l'école, 
au cœur de la grande ville de Marseille, qui fait pendant et écho à la description de Jean le Bleu (" chez les sœurs de la Présentation », Giono, 1972a, p. 11), dans la petite ville de Manosque. La mention de l'école, et de ce trajet, est augurale, elle surgit dès la deuxième phrase du texte : "Tous les matins, "Pov' fille" me menait au pensionnat des sœurs de la Visitation » (Giono, 1977, p. 143). À l'école elle-même est consacré moins d'un paragraphe, dominé par des phrases averbales conférant un caractère impressionniste à ce qui s'apparente de nouveau à une évocation déceptive médiée par les sens, mais aimantée ici par la religiosité du lieu :

J'entrais, et l'odeur d'encens était si forte qu'il n'était plus question d'odeur, mais il était question de bruits et de couleurs. Bruits, d'ailleurs à peine et les mêmes répétés à l'infini, deux ou trois fois : froissements de jupes, claquements de toiles amidonnées, tintements de rosaires, glissements de patins feutrés, craquements de souliers vernis et chuchotements, chuchotements, chuchotements dans l'écho des vastes et hauts couloirs remplis comme d'un énorme et silencieux envolement d'hirondelles. Et couleur simplement d'hirondelles aussi, blancs et noirs, où devait être très extraordinaire la plume de canard sauvage que je portais à ma toque rouge ; blancs des cornettes, noirs des souples soutanes de femmes en grosse laine, et blanc des mains et des visages, et blancs et noirs des grands moellons luisants du parquet. Une lumière rare qui venait des impostes remuait tout ce blanc et ce noir avec de longs bâtons dorés. (Giono, 1977, p. 146)

17 Se démarquant de la référence républicaine, la référence scolaire est ainsi déviée, déformée voire déconstruite par des isotopies dénotant le concret.

Quant aux années de collège, elles font l'objet d'une ellipse explicite dans Jean le Bleu, ou si l'on préfère d'un sommaire très succinct dans lequel seule la situation matérielle et géographique, toute symbolique, de l'établissement intéresse le narrateur: "Je retournais de l'école à quatre heures. J'étais maintenant un élève du petit collège lépreux que la ville avait rejeté là-haut, hors d'elle, sur les aires à blé, du côté des collines " (Giono, 1972, p. 27). L'évaluation axiologique négative opérée par l'adjectif «lépreux»-renforcé par l'adjectif «petit»-, métaphorique et hyperbolique, est également connotée par la mention de la situation excentrée du collège. À la fin du récit cependant, une notation vient s'inscrire en faux contre l'attitude de désintérêt : «Cette fois, c'était bien le rapt. Mon corps était toujours là, dans la ville; c'est lui qui avait quitté le collège et qu'on tenait maintenant dans une banque» (Ibid., p. 168). J. Giono fait ici référence au mois d'octobre 1911, lorsqu'il quitte définitivement l'école pour travailler dans une banque. Le mot « rapt» exprime sans aucune ambiguïté la violence et la douleur de l'événement, un arrachement contraint et prématuré, tandis que la phrase suivante développe l'idée d'un divorce entre le corps et l'esprit, ce dernier restant arrimé au monde scolaire. La netteté de ces formulations vient pour le moins nuancer, voire démentir le caractère assumé et revendiqué, dans les données externes, de la posture d'autodidacte, dénonçant celle-ci comme telle - à moins que l'attachement à l'univers scolaire ne soit soumis à la fiction.

19 Les rares figurations de l'institution scolaire apparaissent pourtant bien dans deux œuvres ayant en commun le caractère autobiographique, et la rattachent à une expérience plus intime qu'intellectuelle ou sociale. Leur caractère évanescent incite alors à chercher les formes de l'inscription des modèles scolaires dans le style littéraire. 


\section{Les styles scolaires : de la phrase simple à la phrase périodique} dichotomie entre un enseignement primaire pour le peuple et un enseignement secondaire pour les élites. Ce dualisme de «deux cultures scolaires qui se trouvent institutionnellement séparées et distinguées à la fin du XIX siècle " (Massol, 2004, p. 270) a été traduit sur le plan linguistique par R. Balibar (1974), qui oppose le français scolaire « national » tel qu'il est enseigné à l'École primaire, privilégiant le style simple, à la langue du secondaire, marquée de l'empreinte rhétorique. Une telle dichotomie relève de la vulgate : "L'emploi du mot propre et de la phrase simple sera ainsi la marque du "français national", quand l'utilisation de la période, des sens figurés et des "latinismes" relèvera des pratiques de l'enseignement secondaire" (Massol, 2004, p. 13). L'ordre secondaire, cependant, n'est pas seulement du côté de l'expressivité, il a longtemps privilégié l'atticisme et l'absence d'effet, qui rejoint la simplicité.

Loin de la prose oralisée et du style simple des romans inauguraux (le cycle de Pan) emblématisé par le titre du premier roman publié, Colline (1928), les tout premiers écrits littéraires de J. Giono manifestent l'influence du genre poétique, genre dominant dans le champ littéraire du XIX ${ }^{e}$ siècle, et dont la transmission est fortement redevable à l'enseignement secondaire. Vers l'âge de seize ou dix-sept ans, J. Giono écrit ainsi des sonnets très classiques, un poème en prose et par la suite divers textes d'inspiration poétique caractérisent sa production au début des années 1920. Resté discret sur ses débuts poétiques pour des raisons idéologiques, s'il n'a pas persévéré dans le genre, du moins l'écrivain a-t-il imposé le registre poétique, en l'acclimatant, comme l'une des principales «marques de fabrique »-avec l'oralité - de sa production romanesque d'avant-guerre :

Ce qui définit le peuple, selon Giono, c'est la poésie comme qualité (et non comme genre), c'est-à-dire une disposition devant le monde, un regard panique ou cosmique. Le «parti pris de poésie » doit être au cœur du roman, afin de montrer les potentialités du peuple, ici du paysan. La "poésie» est ainsi posée comme le critère central d'une littérature prolétarienne. (Meizoz, 2001, p. 333)

À l'échelle de la phrase, c'est l'ordre des mots, dont le respect est central dans l'apprentissage de la grammaire à l'École primaire (Balibar, 1985b), qui est bousculé par le langage poétique au moyen des procédés d'inversion et d'insertion. Or, la question de la norme langagière, et au-delà de la contrainte en général, s'avère au cœur de la problématique scolaire, et tout particulièrement du rapport ambigu de J. Giono à la culture scolaire ${ }^{6}$.

En dépit des affirmations de l'auteur comme de l'indéniable empreinte du français scolaire dans ses premiers romans, où « la simplicité et la redondance syntaxique [...] connotent un récit spontané et naïf ou un exercice de français élémentaire, aux antipodes [...] des finesses narratives du récit littéraire » (Meizoz, 2001, p. 338), il n'est donc pas illégitime de s'interroger sur la présence de traces du français de l'enseignement secondaire dans son œuvre, dans la perspective de réévaluer l'empreinte de la rhétorique, dont on sait par ailleurs que l'influence persiste dans la culture littéraire du lycée au début du $\mathrm{xx}^{e}$ siècle, malgré sa destitution officielle. La littérature critique sur J. Giono a eu tendance à réduire cette empreinte aux tropes, 
voire aux métaphores, dans des premiers romans visant «à contrer symboliquement une parole normée, émanation de la culture savante » (Ibid., p. 363). Mon hypothèse est celle d'un refoulement de la culture scolaire, et en particulier de l'elocutio rhétorique hypothèse qui s'inscrit dans le cadre du champ de recherche plus large sur les modalités de la persistance de la rhétorique après son démantèlement institutionnel. Voyons dans les textes ce qu'il en est de cette séparation théorique à partir de l'opposition entre phrase simple et phrase complexe ${ }^{7}$, qu'informe le double paradigme langue du primaire $v$ s langue du secondaire.

\subsection{La phrase simple stylisée}

Encore au début du $\mathrm{xx}^{\mathrm{e}}$ siècle, la doctrine scolaire reprend les «qualités générales du style (...) qui subsument les genres et dont traitent tous les manuels de rhétorique depuis la première moitié du XIX siècle»(Kaës, 2020, p. 15) ${ }^{8}$. Conformément aux principes de clarté et de pureté notamment, la phrase idéale est de taille moyenne, mais l'évolution de la culture scolaire autour de 1900 érige la phrase brève et simple en modèle?.

Le style primaire, d'après R. Balibar, est fondé sur la pratique de cette phrase simple, liée au culte tout nouveau du "naturel ", et qui est incarnée par un type d'exercice scolaire: "l'essentiel du travail linguistique primaire est la rédaction élémentaire, c'est-à-dire la simple représentation écrite de la simple réalité. La simplicité, facteur d'universalité, résulte elle-même d'un travail spécial de schématisation" (Balibar, 1985a, p. 338). Le corollaire de la simplicité syntaxique est la brièveté de la phrase : ce sont les deux caractéristiques primordiales du français scolaire ${ }^{10}$.

Or, les romans du premier J. Giono se rattachent à ce dernier non seulement par leur langue, mais encore par la prégnance de l'univers naturel - " élémentaire " pourrait-on dire -, "le grand thème scolaire de la représentation de la campagne " (Massol, 2004, p. 266), un topos qui a contribué à cataloguer l'auteur parmi les écrivains régionalistes. La phrase narrative simple et brève, soutenue par le style asyndétique, est repérable dans les romans du cycle de Pan, par exemple dans Colline, où la subordination est très limitée sur le plan à la fois quantitatif et qualitatif : « Il donna un coup de jarret. Il avait tapé comme sur du fer. Il ne monta pas. Il avait de longues lianes d'eau ligneuse enroulées autour de son ventre. Il serra les dents. Il donna un coup de pied. Une lanière d'eau serra sa poitrine. Il était emporté par une masse vivante " (Giono, 1971, p. 203-204). Dans cette séquence où les notations narratives alternent avec des notations descriptives, la discontinuité est battue en brèche par la reconduction du même patron syntaxique $\mathrm{S}-\mathrm{V}-\mathrm{C}$. Dans les quelques cas d'entorses à ce schéma, la position post-verbale est néanmoins saturée par un autre type d'élément (forclusif de la négation, comparatif, complément d'agent). La récurrence du patron surdétermine en l'occurrence la thématisation de l'acharnement et de la résistance. De manière générale chez $\mathrm{J}$. Giono, le style simple est favorisé par l'aspect ponctuel du passé simple dans les passages narratifs, ainsi que par la place importante qu'occupent les dialogues, lesquels suscitent des notations didascaliques : « Matelot se mit à battre le briquet. Il souffla sur l'amadou. [...] Il alluma sa pipe. Il donna l'amadou à Antonio. Antonio souffla » (Giono, 1972, p. 191).

Néanmoins, à y regarder de plus près, dans ce type de séquence la phrase gionienne prend ses distances vis à vis des prescriptions scolaires en matière de style. Certes, le 
souci de l'écueil de la phrase « embarrassée » et " obscure » est une constante dans les manuels ${ }^{11}$. Mais s'il est recommandé d' « éviter les phrases trop longues" (Myard \& Méneret, 1911, p. 252), « lourdes » (Grigaut, 1904, p. 86), il convient dans le même temps de "varier la forme et la coupe des phrases et des propositions» (Ibid., p. 255). "Le moyen de remédier à cet inconvénient c'est de varier le mot initial, de ne pas commencer les phrases invariablement par le sujet, et de donner le plus souvent possible des sujets différents à celles qui se succèdent » (Grigaut, 1904, p. 88). Or, J. Giono fait la part belle à l'homéoptote du pronom personnel sujet immédiatement en tête de phrase. À rebours des recommandations, les séries de phrases ci-dessus instaurent une "uniformité " (Myard \& Méneret, 1911, p. 255) syntaxique et rythmique, une "monotonie» subversive par rapport au modèle de l'École primaire. Mais l'on sait que la phrase scolaire élémentaire est encore biaisée par les traits d'oralité, en particulier le pronom ça et les tournures présentatives (Meizoz, 2001, p. 349-364).

Historiquement, la phrase brève a aussi partie liée avec l'efficacité rhétorique, mais au début du $\mathrm{xx}^{\mathrm{e}}$ siècle, l'«idéal rhétorique de la pointe a cédé devant une diversité plus grande de valeurs [...]-» (Piat, 2009, p. 203). Dans les textes de J. Giono, à la phrase simple en série s'oppose ainsi la phrase simple isolée à valeur conclusive, qui renoue avec cet art de la pointe, acclimaté au texte narratif :

D'abord, Gondran a creusé un trou sous le genévrier le plus touffu, et quand il a atteint la terre noire, il a mis sa bouteille au frais. Il a choisi une bonne branche à l'abri des fourmis pour pendre son carnier, puis, manches troussées, il s'est mis au travail.

Et l'acier de sa bêche a chanté dans les pierres. (Giono, 1971, p. 146)

Associée au rythme de l'alexandrin, la phrase détachée offre peut-être une réminiscence hugolienne, V. Hugo faisant partie des auteurs cités dans les recueils scolaires de Morceaux choisis à l'époque de la scolarité de J.Giono. Quant à la conjonction "et", derrière la valeur de relance, il n'est pas exclu de voir ici un latinisme (sens de "même», "aussi»), créant une superposition de la phrase secondaire et de la phrase primaire.

La brièveté et la simplicité ne constituent cependant pas les seuls critères définitoires de la phrase primaire :

Quelques particularités du style d'école primaire ont pu être relevées. [...] Mais la phrase courte de l'écriture scolaire a besoin d'être relevée, rehaussée, pour se détacher de la banalité de l'oral. Une phrase élégante démarre si possible avec un circonstanciel évocateur, qui plante un décor ou prépare une atmosphère [...]. Le cadre une fois posé, la rhétorique primaire utilise un certain nombre de procédés qui visent à donner une impression de naturel, de spontanéité, de bonhomie, d'enjouement. Les noms, les verbes doivent si possible être accompagnés d'épithètes pittoresques ou convenues, d'adverbes d'intensification: la phrase, même brève, doit donner une impression de plénitude. Le plus efficace, c'est l'exclamation expressive [...]. Autre procédé attaché au style primaire, l'apposition fréquente qui, en ralentissant le rythme de la phrase, lui donne un peu de la pondération et de la noblesse qu'elle s'interdit de chercher dans l'appareil complexe des propositions subordonnées. [...] Dernier point [...], les clichés, les « blancs manteaux », les « blancs linceuls » de la neige, « le soleil dardait ses rayons », « les prés émaillés de fleurs », etc. (Chervel, 2006, p. 711-712; je souligne)

31 Or, J. Giono élimine la plupart de ces caractéristiques de la "rhétorique primaire » seul le circonstant en tête de phrase n'est pas rare - de sa phrase simple, dont la stylisation est interprétable sans doute autant comme symbolisant la pureté élémentaire du monde naturel que comme participant de l'image d'une simplicité 
fruste. Ainsi, à rebours des clichés, les figures imageantes originales qui caractérisent la première manière de l'écrivain détournent la phrase du cadre scolaire élémentaire marquée par le mot propre. L'incipit du Chant du monde est éloquent à cet égard, qui mêle personnification et animalisation hétérogène (je souligne) : «Le fleuve roulait $\grave{a}$ coups d'épaules à travers la forêt, Antonio s'avança jusqu'à la pointe de l'ile. D'un côté l'eau profonde, souple comme du poil de chat; de l'autre côté les hennissements du gué. Antonio toucha le chêne » (Giono, 1972b, p. 189).

Enfin, un autre trait par lequel la phrase gionienne s'écarte du dogme scolaire en matière de phrase brève, est la phrase sans verbe conjugué, qui émaille ses premiers romans: "Tout d'abord, les phrases doivent être complètes au point de vue grammatical : nous voulons dire qu'il faut s'assurer de l'existence du sujet, du verbe, et de tous les autres termes essentiels » (Grigaut, 1904, p. 77). Or, la phrase averbale fait l'objet d'un engouement dans la littérature au début $\mathrm{du} \mathrm{xx}^{\mathrm{e}}$ siècle. Cette influence ou porosité de la production littéraire contemporaine est d'ailleurs confirmée par un autre trait récurrent dans les romans d'avant-guerre, la substantivation des adjectifs, dans le cadre du «style substantif » dont le développement remonte à la seconde moitié du $\mathrm{XIX}^{\mathrm{e}}$ siècle. Ambiguë, la phrase graphique sans verbe se prête donc à deux interprétations concurrentes, l'une en faveur d'une simplicité radicalisée, l'autre dans le sens d'une recherche d'effet littéraire.

En somme, J.Giono radicalise la phrase scolaire primaire sans pour autant la systématiser. En effet, le style de l'écrivain n'est pas homogène du point de vue linguistique, la brièveté et la simplicité dominantes masquant par ailleurs des incursions du côté du style littéraire : «Giono mêle les références culturelles dans une langue où les métaphores insolites, triviales ou précieuses alternent avec les idiomatismes du parler populaire et les expressions provençales francisées » (Thiesse, 1991, p. 143-144). L'ensemble produit ainsi un texte métissé y compris au plan syntaxique interpropositionnel et interphrastique.

\subsection{La phrase périodique}

Avant d'aborder l'autre versant de la phrase, les unités longues, il convient de souligner que culture primaire et culture secondaire ne sont pas totalement étanches, comme l'atteste la phrase simple reconduisant, en le revisitant, l'«idéal rhétorique de la pointe ». Au plan thématique, la continuité entre les deux cultures est assurée par "l'exaltation des beautés naturelles [qui] se retrouve dans la poésie virgilienne, ces extraits des Géorgiques que les lycéens devaient traduire, retenir et, jusqu'en 1880, imiter dans leurs vers latins » (Massol, 2004, p. 266). Si l'absence de figuration de l'art oratoire dans la fiction gionienne est notable, l'hypothèse d'un style périodique dans les romans d'avant-guerre n'est pas historiquement dénuée de fondement du fait que

[...] la rhétorique ne meurt pas du jour au lendemain, malgré sa disparition de textes officiels en 1902 : elle se manifeste aussi bien dans la persistance de sujets de devoirs qui appellent surtout des qualités d'élocution [...]. Les nouvelles dissertations sont également traitées comme des amplifications [...]. Enfin, la formation rhétorique est présente à travers la manière d'écrire des élèves, malgré les critiques officielles de l'amplification et de l'imitation servile. (Houdart-Merot, 1998, p. 69-70) 
postuler l'empreinte rhétorique dans les textes d'après-guerre, qui ne sont plus dominés par la référence au monde naturel, ni par les métaphores originales, interroge davantage.

Rappelons que pour identifier la période, « c'est moins la longueur de la phrase que la hiérarchisation des propositions qui importe, d'où la distinction à opérer entre la période et les "longues phrases qui [sont] seulement des phrases énumératives" " (Albalat, cité dans Piat, 2009, p. 205). En matière de phrase longue et complexe, comme plus largement en matière de rapport à l'École, J. Giono, qui n'a pas étudié le latin, se situe à l'opposé de M. Proust, chez qui « la longueur et le primat de la subordination sont deux propriétés associées à la fois à la langue latine et au modèle périodique " (Kaës, 2020, p. 229) dans sa version ou du moins sa représentation conventionnelle (phrase complexe par subordination). Néanmoins, dans la pratique, le terme période "recouvre des réalités diverses», y compris "des énoncés syntaxiquement indépendants» (Berrendonner, 2017). Ainsi des traces de la période " coupée » asyndétique sont-elles repérables dans Colline à travers les phrases binaires ou ternaires, parataxiques et de dimension modeste, qui ressortissent par conséquent à l'une des formes attestées de la période. On peut y observer en particulier le « rôle du point-virgule dans l'allongement de la phrase », qui «se retrouvait en effet convoqué, dans la transcription des périodes oratoires, pour diviser les unités de souffle - qui recouvraient les unités de la syntaxe» (Piat, 2009, p. 205 et 215). Il peut être associé au deux-points, comme dans l'exemple suivant: «La nuit plus fraîche est comme une promesse sur leurs joues; devant eux se dresse le grand corps de Lure : la mère des eaux, la montagne qui garde l'eau dans les ténèbres de sa chair poreuse " (Giono, 1971, p. 171). Le premier membre expose ici le cadre de la vision qui se déploie après le point-virgule, tandis que la valeur explicative du deux-points assure la cohésion du second membre, avec un effet de clausule porté par la métaphore. Les périodes transposées en contexte descriptif, dans Colline, adoptent donc une forme minorée (Jollin-Bertocchi, 2017) offrant en quelque sorte une image expansée de la phrase simple. En somme, la phrase complexe se coule dans le moule de la phrase simple.

En regard de cette première forme d'appropriation, «la période glisse [...] vers une phrase accumulative - que l'on peut qualifier simplement de phrase longue " (Piat, 2009 , p. 205), forme dérivée typique du $\mathrm{XX}^{\mathrm{e}}$ siècle. Un basculement général des masses phrastiques s'opère dans ce type d'unité où

[...] l'allongement non périodique de la phrase entrâne le déplacement du travail de la zone gauche (l'attaque de la phrase) à la zone droite (la fin de la phrase). Or, le travail d'amplification à l'ouverture de la phrase apparaît plus «logique »: on y attend les éléments destinés à donner un cadre au propos (dont les compléments circonstanciels). [...] De la période rationnelle s'est ainsi dégagée une phrase (simplement) longue, caractérisée par un défaut de structuration et par une recherche tendancielle d'amplification à droite. (Piat, 2009, p. 212 et 217)

Elle s'observe dans les romans lyriques d'avant-guerre, où elle est susceptible de côtoyer la phrase brève dans des séquences descriptives :

Il savait si les brochets sortaient des rives, si les truites remontaient, si les caprilles descendaient $d u$ haut $d u$ fleuve et, parfois, il se laissait enfoncer, il ramait doucement des jambes dans la profondeur pour essayer de toucher cet énorme poisson noir et rouge impossible à prendre et qui, tous les soirs, venait souffler sur le calme des eaux un long jet d'écume et une plainte d'enfant. (Giono, 1972, p. 200)

Pratiques, $191-192$ | 2021 

juxtaposées ou coordonnées, que sur les expansions nominales. Dans Mort d'un personnage, la phrase énumérative prend des proportions plus importantes, ainsi précisément lors de l'évocation du trajet vers l'école :

Nous passions aussi devant un long mur percé de fenêtres sales, derrière lesquelles on entendait des bras de fer brasser une étrange pâte qui sentait le papier et l'encre d'imprimerie; nous passions devant des terrasses de cafés entrouverts, dans lesquels on voyait des femmes de ménage qui promenaient des balais entre des colonnes de chaises cannées, entassées les unes sur les autres, et alors sortait à notre rencontre une odeur de sciure, d'alcools et de tabac qui impressionnait fortement «Pov' fille», car, je sentais sa main se tendre comme la corde quand le vent gonfle le foc; je regardais en haut ses yeux qui se perdaient; puis, nous passions devant la porte qui sentait la toile à sac et le crottin de cheval, et le bras de «Pov'fille» redevenait mou comme la corde d'une voile qui fasseye, puis nous passions devant la boulangerie qui sentait le pain chaud, la boucherie qui sentait le sang sale, le fleuriste qui sentait l'herbe, les cabinets qui sentaient l'urine et une forte odeur de café fumant, les bureaux de la banque qui sentaient également l'urine, les bureaux des hommes d'affaires, des avoués, des huissiers, des commissionnaires, dont les vestibules sentaient également l'urine, plus le crachoir et le cendrier froids, puis les bureaux des exportateurs et des assureurs maritimes qui sentaient l'urine de chien et le soufre qu'on avait répandu contre les bornes des portes cochères pour éloigner les chiens, puis, tout de suite, une boulangerie qui sentait une adorable odeur de brioche chaude, où d'ailleurs « Pov » fille' achetait la brioche de deux sous de mon déjeuner. (Giono, 1977, p. 145)

La structure d'ensemble est fondée sur la réduplication du même schéma syntaxique, renforcée par la reprise lexicale : Sujet («nous ») - Verbe («passions ») - Objet expansé par une subordonnée relative. Dans la seconde partie de la phrase, le procédé d'accumulation se focalise sur la démultiplication du complément d'objet. La plupart des subordonnées y sont des relatives explicatives, soit des équivalents propositionnels de l'apposition adjectivale, ce qui constitue peut-être une manière de concilier la rhétorique de l'École secondaire et celle de l'École primaire. En première analyse, l'accumulation serait interprétable comme une démultiplication de la phrase simple dans un inventaire à la J. Prévert (paru en 1946, soit deux ans avant la publication du roman de J. Giono), proche du pastiche de la rédaction scolaire, récit ou description portant sur les «sujets les plus simples et les mieux connus des enfants » (Chervel, 2006, p. 705). Mais il convient aussi de prendre en compte le fait qu'à l'époque classique, les phrases complexes par enchâssements qui sont données comme des exemples de périodes «contiennent en outre des structures de liste qui leur confèrent un rythme remarquable » (Berrendonner, 2017).

Est-ce à dire que la phrase périodique prototypique est bannie, réduite à la période coupée ou diluée dans la phrase longue accumulative? Pour être très sporadique, la période ample et hiérarchisée n'en émerge pas moins nettement dans Mort d'un personnage, où elle est marquée par l'allongement à gauche qui contribue à la structurer logiquement et rythmiquement, et bornée à droite par un effet de clausule reposant sur une segmentation dans le premier exemple, et par un effet de relance hyperbatique dans le second :

Et, malgré que, dans son âme, avec ces bruits et ces odeurs auxquels se mêlaient les bruits et les odeurs de la mer et les bruits et les odeurs des lointaines collines sur lesquelles brûlait parfois l'incendie balsamique de forêts de pins, elle devait se composer avec le mot ville un monstre prodigieux dont la connaissance nous aurait pétrifiés de stupeur, elle avait la grandeur de tenir compte des vents et du calme et 
de ne pas trop désorienter son visage de l'objet que nous, nous regardions. (Giono, 1977, p. 192)

Mais, tandis que pour les fleurs de la vallée de Saint-Pons ç'avait été seulement pendant le court instant que le bouquet s'était paré dans la mitaine à la pointe des doigts nus sans même que sur ses pentes elle ait tourné la tête, sans même qu'elle ait ralenti sa fuite, ayant gémi ce tout petit gémissement comme un adieu particulier, ici c'était un gémissement de stupeur, puis d'appel comme si, dans ses profondeurs, elle avait été prise d'un doute et qu'elle ait arrêté sa fuite, ne sachant plus si ce qu'elle voulait rejoindre était en bas ou ici. (Ibid., p. 198)

Ces phrases recréent le modèle phrastique de l'enseignement secondaire, forme imbriquant ici l'analyse psychologique et la description. Si l'objet du discours est le personnage de Pauline, l'héroïne féminine du cycle du Hussard, à l'extrême fin de sa vie, personnage d'un temps où régnait encore la rhétorique, Pauline est également, dans ce roman précis, une figuration de la mère illettrée de J. Giono, ce qui est propre à suggérer un effet de grandissement, ou à l'inverse un soupçon d'ironie dans l'appropriation de la forme périodique. Celle-ci resurgit deux pages plus loin, emportée par une vision hugolienne de l'abime en guise de clausule :

Si elle était arrivée avec ses beaux grands yeux, ses épais cheveux d'argent, son petit visage en fer de lance, sa noblesse, la distinction modeste de chacun de ses gestes, dans n'importe quelle assemblée humaine on l'aurait aimée, entourée, servie ; on aurait essayé de la retenir et de la saisir. Si elle s'était assise, à bout de forces, à quelque coin de rue, il y aurait eu cent hommes, cent messieurs pour la prendre sous le bras et l'aider. Si elle avait demandé l'aumône, elle aurait été millionnaire. Mais, lisse et pointue comme un fuseau, lourde et muette comme un plomb, elle échappait à toutes les mains et, quand nous croyions l'avoir saisie, elle glissait entre nos doigts, tombait toujours plus bas, plus profond, plus loin dessous, dans l'enchevêtrement des ténèbres. Nous avions beau tendre la main et nous pencher, nous ne pouvions pas l'atteindre, ou, si on l'atteignait dans un effort où il fallait presque perdre l'équilibre et la vie, c'était pour sentir sa forme polie par la succion de l'abîme glisser de nos doigts et tomber encore plus bas. (Ibid., p. 194)

La segmentation en plusieurs phrases graphiques vise-t-elle à souligner ou à dissimuler le patron périodique ? La longue protase constituée de trois systèmes hypothétiques est rééquilibrée par une apodose de semblable ampleur, formant antithèse avec le premier membre. La rhétorique semble par conséquent constituer un arrière-plan polymorphe qui affleure très ponctuellement dans l'œuvre de J. Giono.

L'imaginaire scolaire qui se fait jour dans l'œuvre de J. Giono à travers la figuration littéraire de l'École et les formes langagières corrobore les déclarations de l'auteur sur sa relation distendue avec l'institution sans toutefois l'entériner tout à fait. La réception des premiers romans, focalisée sur un français moins que scolaire et pis que simple, semble avoir forgé une vision réductrice du style de J. Giono, dont le paradigme rhétorique a été évacué ou du moins borné à une composante restreinte de l'elocutio. Mais à l'examen, surdéterminant une vision périphérique et décentrée, la coexistence des deux versants de la norme scolaire, ces "formations linguistiques antagonistes " (Balibar, 1974, p. 126) que sont le français primaire et le français littéraire secondaire, y est repérable, créant une tension qui a pour effet de liquider l'image de pureté stylistique. Louvoyant entre classicisme et innovation, J. Giono parvient à mimer et à miner les langages et la culture scolaires de l'intérieur au moyen d'un travail sur la phrase qui en offre une réminiscence ambiguë, peut-être vaguement nostalgique. L'obliquité possiblement malicieuse ${ }^{12}$ de la référence à l'École qui caractérise les textes de l'écrivain s'opère en profondeur par le biais d'une forme de distorsion ou de 
réinterprétation des modèles de la phrase, fondés sur la dichotomie historiquement prégnante simplicité/complexité, obérant les possibilités du style littéraire.

D'un point de vue épistémologique, l'approche proposée n'est pas sans présenter certaines limites. Si l'angle stylistique, reposant sur le postulat idéaliste d'un hylémorphisme, s'appuie sur des observables langagiers tangibles, leur identification et plus encore leur interprétation, quelle que soit la rigueur du protocole suivi, laissent place à la subjectivité de l'analyste au sein d'une démarche où la rémanence de la tradition biographique et psychologisante dans les études littéraires n'est pas exclue. C'est pourquoi les interprétations proposées dans cette étude prennent souvent la forme d'alternatives qui sont autant d'hypothèses. De plus, la stylisation et le processus d'« individuation » d'une œuvre se situent autant du côté du récepteur que du côté du créateur, dont l'identité d'écrivain est distincte de son identité civile. Enfin, il est permis de formuler l'hypothèse que l'étude est un tant soit peu biaisée par la vision du chercheur en tant qu'il est lui-même produit d'un système scolaire...

\section{BIBLIOGRAPHIE}

BALIBAR, R. (1974). Les Français fictifs. Le rapport des styles littéraires au français national. Paris : Hachette.

BALIBAR, R. (1985a). L'Institution du français : essai sur le colinguisme des Carolingiens à la République. Paris : Presses universitaires de France.

BALIBAR, R. (1985b). « L'école de 1880. Le français national : républicain, scolaire, grammatical, primaire ». in : Antoine G. \& Martin R. (dir.). Histoire de la langue française 1880-1914. Paris : CNRS Éd., p. 255-293.

BERRENDONNER, A. (2017). « Période ». Encyclopédie grammaticale du français. En ligne : http:// encyclogram.fr/notx/014/014_Notice.php.

BIRON, M. (2007). « Portrait de l'écrivain en autodidacte ». in : Caumartin A. \& Lapointe M.-E. (dirs). Filiations intellectuelles dans la littérature québecoise. @nalyses. En ligne : https:// uottawa.scholarsportal.info/ojs/index.php/revue-analyses/article/viewFile/671/573. BOURDIEU, P. (1979). La Distinction. Paris : Éd. de Minuit.

CARRIÈRE, J. (2000). Jean Giono, du côté de Manosque. Arles : Éd. Harmonia Mundi France.

CHERVEL, A. (1998). La Culture scolaire. Une approche historique. Paris : Belin.

CHERVEL, A. (2006). Histoire de l'enseignement du français $d u$ XVII ${ }^{e} a u$ XX $X^{e}$ siècle. Paris : Éd. Retz.

CiTRON, P. (1995). Giono. Paris : Éd. Le Seuil.

GIONO, J. (1971). « Colline » [1929]. CEuvres romanesques complètes, 1. Paris : Gallimard, p. 127-218.

GIONO, J. (1972a). « Jean le Bleu » [1932]. CEuvres romanesques complètes, 2. Paris : Gallimard, p. 3-186. 
GIONO, J. (1972b). « Le Chant du monde » [1934]. CEuvres romanesques complètes, 2. Paris : Gallimard, p. 189-412.

GIONO, J. (1974). « Noé » [1961]. CEuvres romanesques complètes, 3. Paris : Gallimard, p. 607-862.

GIONO, J. (1977). « Mort d'un personnage » [1949]. Cuvres romanesques complètes, 4. Paris :

Gallimard, p. 143-236.

GIONO, A. (1987). Mon père. Contes des jours ordinaires. Paris : Gallimard.

GIONO, J. (1990). Entretiens avec Jean Amrouche et Taos Amrouche. Paris : Gallimard.

GRIGAUT, M. (1904). Cours de composition française. Enseignement secondaire, primaire supérieur et professionnel. Paris : H. Paulin.

HOUDART-MEROT, V. (1998). La Culture littéraire au lycée depuis 1880. Rennes : Presses universitaires de Rennes.

JOLLIN-BERTOCCHI, S. (2017). «L'instabilité des contours de la phrase dans Colline de Giono : du manuscrit au texte publié ». Giono : le texte en devenir, actes du colloque des 10-12 septembre 2015. Aix-en-Provence/Manosque : Presses universitaires de Provence.

KAËs, E. (2020). Proust à l'école. Genève : Droz.

JEY, M. \& PERRET, L. (dirs). (2020). L'Idée de littérature dans l'enseignement. Paris : Éd. Classiques Garnier.

MASSOL, J.-F. (2004). De l'institution scolaire de la littérature française (1870-1925). Grenoble : Éditions littéraires et linguistiques de l'université de Grenoble.

MEIzoz, J. (2001). L'Âge du roman parlant (1919-1939). Genève : Droz.

MEIZOZ, J. (2007). Postures littéraires. Mises en scène modernes de l'auteur. Genève : Slatkine.

MYARD, J. \& MENERET, L. (1911). Grammaire et composition française. Paris : Delagrave.

PHILIPPE, G. (2003). « Le discours scolaire sur la littérature et le style au début du Xx siècle ». in : Amossy R. et Maingueneau D. (dirs). L'Analyse du discours dans les études littéraires. Toulouse : Presses universitaires du Mirail, p. 379-388.

PIAT, J. (2009). « La Langue littéraire et la phrase ». in : Philippe G. \& Piat J. (dirs). La Langue littéraire : une histoire de la prose en France de Gustave Flaubert à Claude Simon. Paris : Fayard, p. 179-234.

PIRON, A. (1881). Cours complet de littérature. 2. Style : à l'usage des séminaires et des collèges... Paris : V. Lecoffre.

POLIAK, C. F. (1992). La Vocation d'autodidacte. Paris : Éd. L'Harmattan.

POUCET, B. (2012). L'Enseignement privé en France. Paris : Presses universitaires de France.

RAGON, É. (1920 [1889]). Morceaux choisis. Poètes et prosateurs. Paris : J. de Gigord.

ROCHE, A. (1862). Du style et de la composition littéraire. Paris : J. Hetzel.

THIESSE, A.-M. (1991). Écrire la France. Paris : Presses universitaires de France. 


\section{NOTES}

1. Ce point est également développé par J.-F. Massol $(2004$, p. 305) : « [...] tout écrivain est, certes, d'abord un agent de l'institution littéraire qui mène une carrière conditionnée par les lois propres à cette institution, mais aussi un ancien élève, écolier et/ou collégien, lycéen, étudiant. Ses projets, ses œuvres, son style sont donc fondamentalement en rapport (de soumission absolue, acceptation partielle ou refus, dénégation, révolte...) avec un ou plusieurs exercices scolaires qui ont contribué à le doter, à un moment donné, des compétences scripturales qu'il possède de manière experte, pour ne pas dire magistrale. S'il écrit en fonction de sa situation dans le champ littéraire, des tensions qui le constituent et des débats qui y ont cours, il le fait aussi, moins visiblement peut-être, en fonction des apprentissages premiers qui ont été les siens et en fonction d'une évolution des pratiques scolaires qu'il perçoit de manière plus ou moins précise ».

2. Ce champ de recherche est en plein essor comme en témoigne l'actualité éditoriale : Kaës, 2020 ; Jey \& Perret (dirs), 2020.

3. Cet article m'a été inspiré par le projet de M. Jey et de son équipe, dans le cadre du Labex OBVIL : «L'écrivain, l'institution scolaire et la littérature ", ayant donné lieu au colloque «L'écrivain et son école », 23-25 juin 2016, Université Paris-Sorbonne. Je remercie P. Bruley d'avoir attiré mon attention sur ces travaux.

4. Classe de « rhétorique » rebaptisée ainsi en 1902.

5. À des degrés divers; on peut citer plusieurs écrivains de la génération de J. Giono n'ayant pas obtenu le baccalauréat : H. Poulaille, J. Cocteau, A. Malraux.

6. Cette observation est renforcée par le lien avec la culture antique qui nourrit le premier roman, Naissance de l'odyssée, achevé en 1926 mais refusé par Grasset précisément à cause de sa facture littéraire très marquée.

7. J. Giono accordait une grande importance à la phrase : « Employer des mots neutres. Les plus plats font merveille si la phrase est articulée suivant le rythme du drame. C'est dans la phrase que tout est " (Carnet de 1952).

8. Dans les premiers programmes scolaires relatifs à l'enseignement primaire (1888), les programmes de français sont très succincts, énumérant les notions de langue à acquérir et fixant des repères de rédaction simples. L'étude de la langue est absente dans les programmes du secondaire des années 1880-1890, où seule la littérature est citée, assortie par la suite de quelques notions générales (1902). J. Giono a été placé dans une institution religieuse aux tous débuts de sa scolarité. Dans l'enseignement privé, «[1]es programmes sont certes libres, mais cette liberté pédagogique revendiquée se heurte à une contrainte : la nécessité de présenter les élèves à l'examen du baccalauréat ou au concours des grandes écoles, notamment militaires (...). Cela limite, de fait, l'autonomie et la liberté des contenus d'enseignement " (Poucet, 2012, p. 31). À titre d'exemple, É. Ragon, dans l'avertissement de ses Morceaux choisis (1886, plusieurs rééditions jusqu'en 1920), fait explicitement référence aux «programmes de l'enseignement secondaire classique » et à ceux de "l'enseignement secondaire spécial », auxquels il se conforme avec néanmoins quelques libertés.

9. Voir Chervel, 1998, cité dans Kaës, 2020, p. 240.

10. L'injonction à la brièveté répond à une préoccupation qui est moins d'ordre pragmatique que pédagogique et esthétique : «d'une manière générale, les débutants 
feront bien de composer des phrases peu étendues. Toutefois, là encore il ne faut pas d'excès, sinon, le style paraîtrait 'haché' » (Ibid., p. 86).

11. Voir A. Piron (1881), A. Roche (1862).

12. J. Giono était très conscient de son art. Avec Noé (1961), il a signé une autobiographie de son écriture, non dénuée d'humour, qui donne une idée du climat de sa création, conçue comme activité artistique et artisanale où s'affirme le primat de la singularité.

\section{RÉSUMÉS}

L'article examine le rapport problématique que J. Giono entretient avec l'École à travers la construction de la référence scolaire. Après une présentation des données externes relatives à sa «posture» (Meizoz, 2007) de semi-autodidacte, la présence de l'École dans son œuvre est interrogée à travers sa figuration dans les fictions autobiographiques et les formes langagières, plus précisément les patrons phrastiques, la phrase simple stylisée et la phrase périodique. À partir du deuxième tiers $d u$ XIx ${ }^{e}$ siècle, la référence scolaire en matière d'expression est double en effet, elle recouvre un enseignement primaire destiné à l'instruction du peuple, dominé par un idéal de simplicité, opposé à un enseignement secondaire destiné à la formation des élites, et caractérisé par une plus grande expressivité (Balibar, 1974). La réception des premiers romans de J. Giono, focalisée sur un français régional, populaire et oral, a forgé une vision réductrice du style de J.Giono, dont le paradigme rhétorique a été borné à une composante restreinte de l'elocutio. La coexistence des deux versants de la norme scolaire y est néanmoins repérable, surdéterminant une vision périphérique et décentrée qui a pour effet de liquider l'image de pureté stylistique. Entre classicisme et innovation, J. Giono parvient à mimer et à miner les langages et la culture scolaires de l'intérieur au moyen d'un travail sur la phrase qui en offre une réminiscence ambiguë, peut-être vaguement nostalgique. L'obliquité possiblement malicieuse de la référence à l'École s'opère en profondeur par le biais d'une distorsion ou réinterprétation des modèles de la phrase, fondés sur la dichotomie historiquement prégnante simplicité/complexité.

The article examines the problematic relationship that J. Giono has with the School through the construction of the school reference. After a presentation of the external data related to his "posture" (Meizoz, 2007) of semi-autodidact, the presence of the school in his work is questioned through its figuration in the autobiographical fictions and the language forms, more precisely the phrasal patterns, the stylized simple sentence and the periodic sentence. From the second third of the $\mathrm{xIX}^{\text {th }}$ century, the school reference in terms of expression is double in fact, it covers a primary education intended for the instruction of the people, dominated by an ideal of simplicity, opposed to a secondary education intended for the training of the elites, and characterized by a greater expressiveness (Balibar, 1974). The reception of J. Giono's first novels, focused on a regional, popular and oral French, forged a reductive vision of J. Giono's style, whose rhetorical paradigm was limited to a restricted component of the elocutio. The coexistence of the two sides of the scholastic norm is nevertheless detectable, overdetermining a peripheral and decentered vision that has the effect of liquidating the image of stylistic purity. Between classicism and innovation, J. Giono manages to mimic and undermine school languages and 
culture from within by means of a work on the sentence that offers an ambiguous, perhaps vaguely nostalgic or malicious reminiscence.

INDEX

Mots-clés : école, posture, roman autobiographique, rhétorique, phrase, période.

Keywords : school, posture, autobiographical novel, rhetoric, sentence, period.

\section{AUTEUR}

SOPHIE JOLLIN-BERTOCCHI

Université Paris-Saclay, CHCSC, 78047 Guyancourt, France 